approaching the maximum stimulator output and use of a lower level of threshold acceptance allowed for a more precise estimation of their visual cortical excitability. Importantly, given the comparability between stimulus response plots of controls and patients with dementia with Lewy bodies (Fig. 2), ${ }^{1}$ it is unlikely that use of a $25 \%$ cut-off for threshold adversely affected our findings.

Dr Brigo highlights the issue of non-response to the stimulation and that this may be as a result of causes other than insufficient stimulation strength. Indeed, phosphene perception, or lack of, may not necessarily originate in the visual cortex but may depend on higher visual areas or indeed non-visual areas as well as recurrent processing. ${ }^{2,3}$ However, the reasons that Dr Brigo presents to explain the non-response - including imprecision in finding the optimal position for stimulation delivery over the occiput, greater depth of the primary visual cortex leading to reduced magnetic field strength at the level of the cortex, and use of the figure-of-eight coil - are actually arguments supporting the assumption that failure to respond in some individuals is due to insufficient current stimulation to the neural locus responsible for phosphene elicitation.

In our study we sampled nine equally spaced scalp sites, giving good symmetrical cover of the occiput; this was a compromise between precision and limiting the experiment duration in a vulnerable patient group. The figure-of-eight coil has been frequently used in phosphene research (e.g. Kammer et $a l^{4}$ ) and was chosen because of its spatial accuracy; larger, diffuse-field coils could theoretically activate areas external to the visual areas of interest or indeed induce retinal phosphenes. In addition, we would contend that the transcranial magnetic stimulation (TMS) methodologies we employed meant that we had comparable and, in some cases, better rates of phosphene response compared with other studies in young healthy individuals.

Dr Brigo indicates potential differences in the lower and upper visual cortical activation with TMS and certainly our data of greater phosphene elicitation in the lower visual fields supports this. Our use of the adjusted phosphene threshold ratio to control for group differences in atrophy also accounted for skull thickness, although whether the positions we chose for these measurements directly related to the precise locus of stimulation on the visual cortex, we agree, is a methodological limitation. The use of magnetic resonance-guided stereotactic coil placement, for example, would help with this issue and allow for more precise threshold determination.

As suggested by Dr Brigo we performed an analysis only on those participants who responded to TMS (controls, $n=17$; patients, $n=17$ ) and the findings were in line with our main analyses: there were no significant differences between the controls and patients for phosphene threshold (controls: median 64.0\% $(\mathrm{IQR}=32.5 \%)$; patients: median $67.0 \% \quad(\mathrm{IQR}=20.0 \%)$; $U=139.5, \quad P=0.87$ ) and phosphene response rate (controls: median $6.0(\mathrm{IQR}=7.0)$; patients: median $8(\mathrm{IQR}=5) ; U=112.5$, $P=0.27$ ). Correlations between the Neuropsychiatric Inventory hallucinations subscale score in patient responders and the phosphene excitability measures (phosphene threshold, Kendall's $t=-0.28, P=0.15$; phosphene response rate, $t=0.46, P=0.02$ ) were in the same direction as the main analysis, although less significant owing to the smaller sample and the fact that the four patients who did not respond to TMS at the maximum stimulator output had significantly less severe and frequent visual hallucinations compared with patient responders (Mann-Whitney $U$-test $16.5, P<0.001)$. Clearly, the lack of phosphene response (regardless of cause) is associated with fewer visual hallucinations and thus we would argue that inclusion of non-responders in our analyses is essential in providing a more holistic understanding of the underlying aetiology of this symptom in dementia with Lewy bodies.

1 Taylor JP, Firbank M, Barnett N, Pearce S, Livingstone A, Mosimann U, et al. Visual hallucinations in dementia with Lewy bodies: transcranial magnetic stimulation study. Br J Psychiatry 2011; 199: 492-500.

2 Taylor $\mathrm{P}$, Walsh $\mathrm{V}$, Eimer $\mathrm{M}$. The neural signature of phosphene perception. Hum Brain Mapp 2010; 31: 1408-17.

3 Fried PJ, Elkin-Frankston S, Rushmore RJ, Hilgetag CC, Valero-Cabre A. Characterization of visual percepts evoked by noninvasive stimulation of the human posterior parietal cortex. PLOS One 2011; 6: e27204.

4 Kammer T, Beck S, Erb M, Grodd W. The influence of current direction on phosphene thresholds evoked by transcranial magnetic stimulation. Clin Neurophysiol 2001; 112: 2015-21.

John-Paul Taylor, Institute for Ageing and Health, Newcastle University, Campus for Ageing and Vitality, Newcastle upon Tyne NE4 5PL, UK. Email: john-paul.taylor@ncl.ac.uk; Michael Firbank, John O'Brien, Institute for Ageing and Health, Campus for Ageing and Vitality, Newcastle University, Newcastle upon Tyne, UK

doi: $10.1192 /$ bjp.200.4.345a

\section{Creativity and mental disorder}

Kyaga et al found an intriguing association between creativity and severe mental disorder. ${ }^{1}$ The study draws its strength from a large sample size. However, the retrospective data collection methodology brings with it certain inherent limitations, which the authors have acknowledged, and causal links have been hinted at in the discussion. We would like to bring to attention two issues. First, the role of potential confounders in selection of occupation has not been taken into consideration. The type of occupation one pursues is governed by multiple factors in addition to personnel interest, including educational qualification, opportunity, awareness, location of the job, financial remuneration, familial and other social commitments. ${ }^{2}$ Many of these variables are likely to be affected by the psychiatric illness, although they are modifiable by many independent factors as well. Hence the occupation choices of both individuals with mental illness and their children (and other family members) are likely to be affected by many variables which need to be taken into consideration when interpreting Kyaga et als findings.

Another relevant issue for consideration is the way occupation is defined in their study. The definition of occupation used in (mental) health studies has been criticised for being too restrictive. ${ }^{3}$ National descriptions of occupation tend to classify only those occupations that have economic relevance. ${ }^{4}$ Such an approach is likely to miss someone employed as a labourer who paints during their leisure time or to miss certain population groups. For example, in many settings the majority of women are likely to be the primary caregiver (i.e. housewife) and not formally 'employed'. Future studies could be strengthened by the use of a more comprehensive and inclusive definition of occupation.

1 Kyaga $\mathrm{S}$, Lichtenstein $\mathrm{P}$, Boman $\mathrm{M}$, Hultman $\mathrm{C}$, Långström $\mathrm{N}$, Landén $\mathrm{M}$. Creativity and mental disorder: family study of 300000 people with severe mental disorder. Br J Psychiatry 2011; 199: 373-9.

2 Llena-Nozala A, Lindebooma M, Portrait F. The effect of work on mental health: does occupation matter? Health Econ 2004; 13: 1045-62.

3 Townsend E. Occupation: potential for personal and social transformation. J Occup Sci Aust 1997; 4: 18-26.

4 Human Resources and Skills Development Canada. National Occupational Classification (NOC) 2011: Occupational structure. HRSDC, 2012.

Bichitra N. Patra, Post Graduate Institute of Medical Education and Research (PGIMER), Chandigarh, India. Email: patrab.aiims@gmail.com; Yatan Pal Singh Balhara, Department of Psychiatry and De-addiction, Lady Hardinge Medical College and Smt. Sucheta Kriplani Hospital, New Delhi, India

doi: 10.1192/bjp.200.4.346 